\title{
Acompañamiento terapéutico para la aceptación o rechazo de una prótesis: Caso de paciente infantil con amputación de miembro superior izquierdo
}

\section{Therapeutic accompaniment for the acceptance or rejection of a prosthesis: Case of a child patient with amputation}

\author{
MARTINEZ-TELLEZ, María $\dagger^{*}$, MARTINEZ-TELLEZ, Rubelia, SORIANO-PORRAS, Dulce y \\ APAN-ARAUJO, Karla
}

Universidad Politécnica de Amozoc

ID $1^{\text {er }}$ Autor: María, Martínez-Téllez / ORC ID: 0000-0002-2816-1362, Researcher ID Thomson: H-2293-2019, arXiv Author ID: XYQZYD-DA44HZ

ID $1^{\text {er }}$ Coautor: Rubelia, Martínez-Téllez, ORC ID: 0000-0003-0164-066X, Researcher ID Thomson: E-8229-2018, arXiv Author ID: GYNFU8-XHIM03

ID $2^{\text {do }}$ Coautor: Dulce, Soriano-Porras / ORC ID: 0000-0001-7398-0693, Researcher ID Thomson: E-8233-2018, arXiv Author ID: 8GOGIR-3HFFHH

ID $3^{\text {er }}$ Coautor: Karla, Apan-Araujo / ORC ID: 0000-0003-3373-226X, Researcher ID Thomson ID: E-8338-2018

DOI: $10.35429 / J P .2019 .10 .3 .1 .13$

Recibido 19 de Octubre, 2019; Aceptado 29 Noviembre, 2019

\begin{abstract}
Resumen
El proyecto designado con el nombre "Acompañamiento Terapéutico para la Aceptación o Rechazo de una Prótesis: caso de paciente infantil con amputación de miembro superior" se desprende de la investigación denominada Adaptación y Habilitación Fisioterapéutica de Prótesis Articulada Mioeléctrica de Mano, el cual fue un trabajo multidisciplinario y multinstitucional, donde la construcción, diseño adaptación y habilitación incluyeron a las áreas de manufactura, electrónica, programación y terapia física. El área de la psicológica se estructuró trasversalmente a la adaptación-habilitación fisioterapéutica como respuesta a la necesidad de identificar los factores que estaban influyendo en la aceptación o rechazo del uso de la prótesis. El objetivo del proyecto fue identificar el conjunto de representaciones discursivas que favorecen el rechazo o la aceptación de la protesis utilizando las técnicas de terapia narrativa y terapia de juego a través de la entrevista semiestructurada. El diseño metodológico es análisis de caso, el cual es un tipo de estudio de corte exploratorio y cualitativo. Los resultados obtenidos de la identificación de las representaciones permitieron proponer un protocolo de intervención para acompañar a pacientes de 4 a 12 años candidatos a usar una prótesis.
\end{abstract}

Representaciones, Acompañamiento Terapéutico, amputación, Prótesis mioeléctrica, Toma de decisión

\begin{abstract}
The project named "Therapeutic Accompaniment for the Acceptance or Rejection of a Prosthesis: case of a child patient with amputation of the upper limb" is derived from the investigation called Adaptation and Physiotherapeutic Qualification of Handheld Myoelectric Prosthetic Prosthesis, which was a work multidisciplinary and multi-institutional, where construction, design adaptation and habilitation included the areas of manufacturing, electronics, programming and physical therapy. The psychological area was structured transversally to physiotherapeutic adaptationhabilitation in response to the need to identify the factors that were influencing the acceptance or rejection of the use of the prosthesis. The objective of the project was to identify the set of discursive representations that favor the rejection or acceptance of the prosthesis using the techniques of narrative therapy and game therapy through the semi-structured interview. The methodological design is case analysis, which is a type of qualitative and exploratory study. The results obtained from the identification of the representations allowed us to propose an intervention protocol to accompany patients of 4 to 12 years candidates to use a prosthesis.
\end{abstract}

Represetations, Therapeutic accompaniment, amputation, Myoelectric prothesis, Decision making

\footnotetext{
* Correspondencia del Autor (maría.martinez@upamozoc.edu.mx)

$\uparrow$ Investigador contribuyendo como primer autor.
} 


\section{Introducción}

Según el Instituto Nacional de Estadística y Geografía existen cerca de 780 mil amputados en México, es decir, por año hay más de $27 \mathrm{mil}$ personas que han perdido algún segmento del cuerpo y que no son atendidos para su rehabilitación o para el uso de prótesis, lo cual representa un problema económico y de salud. (citado por Academia Nacional de Medicina, 2015).

La problemática de salud se especifica en las posibilidades de acceder a oportunidades de empleo, a espacios de ocio y entretenimiento, generando un estrato de población marginada. Para resolver la problemática, se ha buscado que, en los procedimientos de amputación- también denominadas cirugías no conservadoras- al momento de retirar de manera total o parcial el segmento del miembro afectado, el muño que se construye después del procedimiento tenga las propiedades necesarias para sustituir la parte perdida con un injerto o una prótesis. Echavarren (como se citó en Cabrera; 2012, 3).

Sin embargo, el proceso de amputación y adaptación de una prótesis no son fáciles, puesto que sus efectos están asociados al dolor del miembro fantasma, a la compensación muscular para la protección corporal (Martínez, Trujeque, Soriano y Apan, 2018). Así como efectos psicosociales referentes a la representación del cuerpo normal y funcional, lo cual la persona se puede topar con desigualdad en el acceso a oportunidades de desarrollo y rechazo social (Organización Mundial de la Salud [OMS], 2017).

Por otro lado, el tiempo de latencia de las nuevas estructuras de la imagen corporal, son resueltas por el propio paciente, sin tener un acompañamiento psicoterapéutico, ya que los protocolos de actuación siguen estando centrados en resolver las dolencias del cuerpo físico, dejando en un segundo término el cuerpo imaginario y social (Academia Nacional de Medicina, 2015)

Aunado a esta problemática los protocolos usados por las disciplinas psi se han centrado en brindar contención, sostén, resiliencia pre y post operatoria con el propósito de permitirle a las personas manejar el nuevo panorama (Protesis Avanzadas, 2019).
Por esta razón, el objetivo general de esta investigación es: Identificar el conjunto de representaciones discursivas que favorecen el rechazo o la aceptación de la protesis a través de la entrevista semiestructurada, terapia narrativa y la terapia de juego. Así como, conocer las representaciones de la imagen corporal, el género, y la etapa de desarrollo como elementos que favorecen la toma de decisión para el uso de una protesis. Con el propósito de proponer un protocolo de intervención con pacientes de edad de 4 a 12 años.

\section{Planteamiento del problema}

El presente trabajo tiene el objetivo de proponer un protocolo de intervención psicoterapéutica ad doc a la etapa de desarrollo de pacientes que han sido sometidos a una cirugía no conservadora, (amputación) y que estén con la expectativa a recibir una protesis.

Dentro de la revisión bibliográfica se ubicaron diversas investigaciones y estudios sobre el tema de la amputación, su rehabilitación y la adaptación de una protesis.

En el 2013 en Murcia España realizaron un estudio sobre el sufrimiento de personas amputadas desde un enfoque etnográfico con aplicaciones psicoterapéuticas, es una investigación de corte cualitativo y humanista con el objetivo de describir el sufrimiento y las estrategias de afrontamiento ante la amputación, analizando la aparición de discursos resilientes para establecer la importancia de la ayuda psicológica en los diferentes momentos que los pacientes estén experimentando (Diaz, Leal, Gómez, 2013)

En la Ciudad de México en el Instituto Mexicano de Oncología hicieron un estudio sobre los aspectos psicológicos en pacientes sometidos a un proceso de amputación por enfermedad oncológica con el propósito de identificar los efectos de la intervención psicológica, se encontró que el $40 \%$ de pacientes amputados mostraron de manera favorable la aceptación y adherencia al tratamiento psicoterapéutico, así como, herramientas de afrontamiento ante la amputación (Meingüer, Martínez, Galindo y Rojas, C., 2014)

Font y colaboradores realizaron una revisión sistemática de la literatura sobre los factores psicosociales implicados en la amputación.

MARTINEZ-TELLEZ María, MARTINEZ-TELLEZ, Rubelia, SORIANO-PORRAS, Dulce y APAN-ARAUJO, Karla. Acompañamiento terapéutico para la aceptación o rechazo de una prótesis: Caso de paciente infantil con amputación de miembro superior izquierdo. Revista de Fisioterapia. 2019 
Rastrearon 18 artículos de diferentes países, con metodologías diversas, el propósito de identificarlos fue para mejorar la práctica clínica dirigida a las personas que sufrieron una cirugía no convencional (Font, Llaurado, Pallares y Harcía, 2016). En el mismo año, en Cuba se publica una artículo que propone un protocolo de actuación en la rehabilitación de pacientes amputados de miembro inferior, el cual busca brindar un tratamiento global que considere procedimientos dinámicos y continuos desde la lesión hasta el uso de la protesis, sin embargo, este protocolo solo se centra en el tratamiento anatómico funcional del cuerpo, menciona la importancia de la intervención psicológica, pero el detalle y rigurosidad de los procedimientos médicos son el centro del tratamiento (Goventes, Alba y Arias, 2016).

Se identificó una investigación más que propone brindar herramientas psicoterapéuticas para la intervención psicológica en el momento previo a un procedimiento quirúrgico no conservador. Plantea el uso de la técnica psicoterapéutica denominada psicoprofilaxis, con la finalidad de preparar emocionalmente a los infantes y a la familia que permitirá un espacio de afrontamiento con la menor cantidad de secuelas psicológicas y físicas posibles, dentro de un abordaje preventivo de salud. Puesto que cualquier procedimiento quirúrgico en los infantes constituye una fuente de estrés psicológico, que obliga al paciente a enfrentarse a situaciones desconocidas (Miranda, 2017)

Por lo tanto, al analizar el contenido y propuestas en la revisión bibliografía, se observa que la problemática inicia al momento de no encontrar protocolos de intervención que puntualicen no solo lo tratamientos pre y post operatorios, sino lo escaso de los elementos que le permitan a los médicos y los terapeutas físicos, solicitar, colaborar y priorizar el acompañamiento psicológico antes y durante la habilitación y adaptación de una protesis.

Finalmente, tanto la revisión bibliográfica y el presente estudio evidencian la problemática metodológica y técnica en la intervención con pacientes amputados. Ya que durante el proceso terapéutico del caso que se describe a continuación, llegó a identificar que el paciente solo fue atendido desde los quehaceres médicos, es decir, solo se tuvo en cuenta el cuerpo anatómico, orgánico y funcionalidad.
Esto llevo a que la atención psicológica se tomara en cuenta hasta que se manifestaron conductas de confusión, indecisión, rechazo, ira en el paciente, y en la familia se observara indiferencia en el proceso de la adaptación y habilitación de la prótesis, un resultado no favorable para el proyecto de protesis mioeléctrica. Es por ello por lo que él, la necesidad de identificar los factores que favorecen el rechazo o aceptación de una prótesis tiene como propósito establecer un protocolo de intervención psicoterapéutica a la par de los tratamientos médicos $\mathrm{y}$ fisioterapéuticos, que permitirá en un futuro, fomentar el trabajo interdisciplinario.

\section{Diseño Metodológico}

El diseño metodológico clínico es de índole exploratorio y cualitativo, el cual busca describir de manera detallada las dimensiones que componen un fenómeno determinado.

\section{Método}

El método utilizado fue el análisis de caso que se basa en la descripción y observación de la realidad, tal como la percibe el sujeto o sujetos de estudio, por ejemplo, saber cómo el paciente percibe el accidente que generó la pérdida del miembro corporal, como describe el sistema de convivencia familiar, escolar, entorno al uso de la prótesis (Hernández, 2003).

El propósito del método de análisis caso es proponer un protocolo de intervención terapéutico para pacientes con amputaciones y/o candidatos a recibir entrenamiento fisioterapéutico para la habilitación y adaptación de prótesis, pues este método permite establecer los límites entre el fenómeno a estudiar y sus variables contextuales, puede ser utilizando para analizar un caso único o múltiples casos a la vez. Yin (como se citó Martínez, 2006,174)

\section{Técnicas}

Las técnicas que fueron aplicadas durante el proceso de trabajo psicoterapéutico fueron la terapia narrativa y la terapia de juego. El objetivo de la terapia narrativa es articular la historia del sujeto a través de su construcción oral, escrita o gráfica, por medio de metáforas, fantasías, que permitirán elaborar nuevos significados de lo vivido, generar alternativas de solución y herramientas para su ejecución (Campillo, 2019).

MARTINEZ-TELLEZ, María, MARTINEZ-TELLEZ, Rubelia, SORIANO-PORRAS, Dulce y APAN-ARAUJO, Karla. Acompañamiento terapéutico para la aceptación o rechazo de una prótesis: Caso de paciente infantil con amputación de miembro superior izquierdo. Revista de Fisioterapia. 2019 
Brinda contención y protección al niño, niña o adolescente que ha sufrido un trauma. Para que se desarrolle con facilidad la narrativa de los hechos dolorosos, es necesario establecer un contexto ordenado y confiable, para que de manera paulatina comprenda que hay opciones y que existen otras maneras de vivir. Ya que el hablar de los hechos traumáticos y las secuelas que se pudieron generar en las diferentes esferas del niño, puede hacer surgir angustia, enojo o tristeza. (Stracalli, 2018)

Por otro lado, la terapia de juego se basa en la observación y construcción de un espacio ludio para el niño, niña o adolescente. La importancia del juego en la etapa de desarrollo infantil y adolescente que comprende de los 4 años a los 12 años es primordial, ya que le brinda la posibilidad de solucionar problemas, construir relaciones con el mundo de los adultos, es la vía para el desarrollo motriz, cognitivo y social.

El juego invita a la construcción del pensamiento causal de las acciones y las conductas, donde los impulsos desbordantes adquieren límites. Es el espacio simbólico para abordar temas complejos como violencia familiar, abuso sexual, dudas sobre sus gustos, problemas familiares, acontecimientos traumáticos que, por la cualidad de abstracción de estos, el juego es el medio para aterrizarlo al lenguaje y estructuras de pensamiento del infantil. (Schaefer, 2012).

Según Nickerson la importancia de trabajar el juego a nivel terapéutico es que el adulto puede entender el mundo del niño de manera natural, permitiendo una mejor relación entre el adulto y el niño, a través de actividades de lúdicas, a diferencia de una conversación abstracta (1973 como se citó en West, J. 2000,15).

Por lo tanto, el propósito de la terapia narrativa y la terapia de juego es construir espacios físicos, imaginarios y simbólicos donde el niño, la niña, el adolescente pueda expresar, identificar y comprender la situación por la que está pasando, es preciso puntualizar que un suceso traumático se puede interpretar por parte del paciente como algo que no hay que decidir, algo que se pude superar por si solo con el tiempo y que es responsabilidad del afectado resolverlo (Flesler, 2011).

\section{Instrumentos}

El instrumento que se utilizó fue la entrevista, la cual es una conversación coloquial, a diferencia de la aplicación de prueba, escalas y cuestionarios, el dinamismo de la conversación permite obtener información profunda, da la oportunidad de aclarar dudas, además este instrumento es utilizado en estudios descriptivos y exploratorios, es una herramienta cualitativa para recabar datos (Diaz, Torruco, Martínez y Valera, 2013)

\section{Desarrollo}

El dispositivo psicoterapéutico con un infante o adolescente está estructurado en un proceso inclusivo, el cual, implica dentro de las sesiones terapéuticas: entrevistas semiestructuradas con los padres, entrevistas, narrativa y juego con el niño o niña y si son necesarias interconsultas con escuela, medico, u otros familiares que estén involucrados en el desarrollo del menor. (Caballo, y Simón, 2002) Este procedimiento estuvo integrado de la siguiente manera:

1. Entrevista semiestructurada con Fisioterapeutas antes del contacto con el paciente: se indagó el motivo de la canalización, descripción de la problemática, las expectativas en relación con el tratamiento fisioterapéutico, la percepción de la relación de la familia con el niño, y cambios comportamentales observados en relación con el tema de la prótesis.

2. Entrevista semiestructurada a los padres: Dentro de los protocolos psicoterapéuticos con infantes, la información que puedan brindar los padres sobre la situación del hijo o hija es importante ya que son ellos los conviven, protegen $\mathrm{y}$ fungen como figuras de autoridad dentro del desarrollo del niño, niña o adolescente. Este procedimiento se realiza con entrevistas previas y de seguimiento. La entrevista inicial fue para indagar la percepción que tienen sobre la situación por la que está pasando el paciente, expectativas sobre el tratamiento de atención psicológica, expectativas sobre el entrenamiento fisioterapéutico y la prótesis, dudas sobre la dinámica de las sesiones y de su participación en el trabajo psicoterapéutico. 
Las entrevistas de seguimiento se llevan a cabo siguiendo las siguientes condiciones: 1) según el avance del niño en el espacio psicoterapéutico; 2) la transferencia e información sobresaliente que se manifieste durante el dispositivo.

3. Sesiones con entrevistas semiestructuradas con el Niño o Niña (paciente): El objetivo de las primeras sesiones denominadas entrevistas, con el niño, tienen el objetivo de establecer el vínculo transferencial, puesto que, sin este, la inserción del dispositivo narrativo-lúdico no se puede concretar. En estas entrevistas se le preguntaron sobre gustos, juegos favoritos, las cosas que no le gustan, se observa la reacción a diferentes estímulos como elección de juegos en específico, manipulación de las reglas, creación de fantasías, percepción de las razones que el cree, del por qué lo llevaron con el psicoterapeuta. En este momento previo se evita abordar el tema, solo que el paciente lo mencione, es como se inicia el trabajo.

4. Entrevistas a los padres de seguimiento: A través de la técnica de terapia narrativa se busca que los padres construyan la historia del hijo o hija, con la finalidad de observar áreas de oportunidad, percepción de los padres sobre la situación traumática, expectativas sobre el desarrollo y futuro del hijo o la hija.

Las entrevistas se estructuraron en tres etapas; la primera fue la construcción de la historia, desarrollo social, emocional y físico, relaciones afectivas dentro de la familia, la escuela y comunidad.

La segunda etapa fue indagar sobre la percepción del accidente, la construcción de la historia del trauma y las representaciones posteriores.

Finalmente, la tercera etapa, analizo la evolución en el tratamiento, cambios de comportamiento, surgimiento de nuevos temas a partir del dispositivo.
El proceso del dispositivo lúdiconarrativo se estructura dialécticamente, puesto que la persona se integra y consolida como sujeto a través de cadenas de discursos entremezclados, los cuales permiten la operación de conductas, decisiones y formas de relacionarse, (Potter, 2008), por lo tanto, el dispositivo usa la entrevista semiestructurada como herramienta central, con el propósito de ir construyendo el universo de las representaciones que están entorno a la fenómeno clínico, es en este caso, la aceptación o rechazo de una prótesis, a través de una visión psicosocial.

La visión psicosocial busca observar los hechos y sus relaciones desde una lectura ternaria, conciben la relación sujeto- sujeto dentro de una tercia de relación donde el sujeto interactuar con un objeto y un alter, este último son las creencias entorno al sujeto, al objeto y a la relación de estos (Moscovici, 2008, 21)

\section{Resultados}

Los resultados obtenidos en el proceso clínico se puntualizan en las siguientes conversaciones ${ }^{1}$, las cuales están divididas en: 1) la historia del paciente y el motivo de consulta; 2) la relación de la prótesis, la mano y el juego; 3 ) el juego, en el discurso paterno y materno; 4) el discurso materno y paterno entorno al género; y 5) la percepción de la prótesis en el discurso materno.

\section{La historia del paciente y el motivo de consulta}

A manera de contexto, a los 8 años el paciente A sufre un accidente en un juego mecánico, que llevo a la perdida de la mano izquierda, le realizaron cirugías para intentar recuperar el miembro afectado, pero terminan amputando, por riesgo a una sepsis que comprometía su bienestar. Es canalizado al departamento de psicología para tratar el proceso de duelo y a terapia física para rehabilitar $\mathrm{y}$ hacerse independiente ante el nuevo panorama. Unos meses más tarde, a su familia le ofrecen entrar a un proyecto de diseño, construcción y adaptación de un prototipo de prótesis Mioelectrica, para Miembro Superior por parte de la Universidad Politécnica de Amozoc.

\footnotetext{
${ }^{1}$ Cabe aclarar que no se transcribieron todas las conversaciones de todo el proceso clínico, solo las más significativas y que estuvieran en relación directa con el tema de la prótesis, el accidente y el juego.

MARTINEZ-TELLEZ, María, MARTINEZ-TELLEZ, Rubelia, SORIANO-PORRAS, Dulce y APAN-ARAUJO, Karla. Acompañamiento terapéutico para la aceptación o rechazo de una prótesis: Caso de paciente infantil con amputación de miembro superior izquierdo. Revista de Fisioterapia. 2019 
El propósito del proyecto de prótesis era diseñar, construir y habilitar una mano robótica, la cual, su movilidad estuviera a cargo de la reestructuración cognitiva de esquemas motores desde el pensamiento para reactivar la motricidad fina y gruesa, utilizando el síndrome de miembro fantasma. El primer modelo que el usaba en algunos momentos del día, era solo una pinza, pero se visualizaba, una mano: estructurada, con falanges, falanginas $y$ falangetas, rotación de muñeca, prensión fina, gruesa y movimiento independiente en cada dedo. Así que la terapeuta física y la maestra en fisiología de la universidad realizaron una serie de rutinas terapéuticas para regresar la movilidad cognitiva de la mano amputada.

Dentro de las sesiones fisioterapéuticas se observaron resistencias a usar el primer modelo de prótesis, por lo tanto, solicitan la canalización a psicología para saber los motivos de la resistencia, los elementos que estructuran la percepción sobre la prótesis y la dinámica familiar.

El Paciente A de 10 años, es el segundo de cuatro hermanos, proveniente de una familia campesina, de clase media baja. Su padre de 70 años se dedica al campo, su madre de 45 años se dedica a la costura, a las labores del hogar, y a atender una papelería.

Él llega consulta cuando estaba terminado el tercer grado de primaria, le gustan las materias de español, de ciencias naturales, pero no las matemáticas, ya que le cuesta trabajo las divisiones. No le gusta dibujar, ni hacer trazos o cosas con colores.

Los programas favoritos del paciente son: Dragón Ball, porque los personajes cada vez que se mueren obtienen súper poderes. Los programas de YouTube con temas variados como bromas pesadas, historias paranormales y video juegos. Sus espacios de juego favoritos son la bicicleta, los coches, la tableta y hacer bromas pesadas.

Los temores frecuentes y aprendidos a través de la dinámica cultural de la comunidad son a las brujas, porque se quitan y ponen las piernas, y se chupan a los niños, al duende, que no tiene cabeza, a la llorona, porque no tiene pies y se lleva a los hombres, y al muerto, que solo grita, pero no se ve.

\section{La prótesis, la mano y el juego}

La primera vez que llega el paciente a sesión se le invita a jugar, el refiere que, ya no es un niño para jugar y cito: "Yo no juego a nada, eso es de niñitos, de niñitos de mami".

Las primeras sesiones él buscaba hablar y no hablar de la mano y de la prótesis, sin embargo, se observaba resistencia de tocar el tema y de jugar. Pero cada vez que entraba al consultorio, iba a lo juguetes, a las marionetas, a los juegos de mesa, a los carritos o llevaba a escondidas, juguetitos, como cochecitos, patinetas, tazos, trompos, lo curioso de estos juegos es que se manipulan con las manos, pero cuando se le invita a jugar huía con la misma frase: "Yo no juego, eso es de niñitos".

Por lo tanto, se toma la decisión de hacer a un lado el objetivo de consulta y plantearle que el venir a consulta era para construir una historia, la que él quisiera y, jugar a lo que le gustaba y hablar de muchas cosas que no forzosamente tenían que ser sobre la mano, el accidente o la prótesis, al informarle de este cambio, su reacción fue la siguiente:

\footnotetext{
Paciente (P): ¿No tengo que hablar de esto? - levantando el muño-

Psi (Yo): No, solo que quieras y salga el tema, se hablara de eso.

P: Puedo hablar de lo que yo quiera... iQue alivio! suspirando hondo, y jorobando el cuerpo- ¿podemos ahora si jugar?

Yo: Si, y a que quieres jugar

$P:$ ¿a lo que tú quieras?

Yo: Toma el juego que gustes.
}

(Transcripción directa sesión 6: abril 2015)

El juego transitó de la siguiente manera, primero tocaba las marionetas se le invita a usarlas, agarraba el Jenga, así cada sesión. Hasta que se toma de decisión de que el psicoterapeuta comienza el juego usando las marionetas utilizándolas para jugar de manera indirecta, Es ahí cuando el paciente A toma tres marionetas una foca, un pez llamado corcel y un pollo, la forma en que se acercó a los juguetes fue cuando la psicoterapeuta voltea para acercar un tapete.

El juego muestra el uso de trampas, no sigue las reglas o las cambia a su favor, pero si estas perjudican a la psicoterapeuta las restablece como estaban. Se mantiene en la demanda de que se le diga que hacer, hasta que, él inicia una conversación con la marioneta de la psicoterapeuta a la cual la nombra loquillo.

MARTINEZ-TELLEZ, María, MARTINEZ-TELLEZ, Rubelia, SORIANO-PORRAS, Dulce y APAN-ARAUJO, Karla. Acompañamiento terapéutico para la aceptación o rechazo de una prótesis: Caso de paciente infantil con amputación de miembro superior izquierdo. Revista de Fisioterapia. 2019 
P: Este loquillo está loco y no me entiende, no me hace caso, así que evolucionare en Foca.

Yo: "Vas a evolucionar enfoca" - en la caja había unos lentes de alambrón pequeños- "entonces te doy estos lentes para que enfoques"

P: "Asch, no quiero los lentes, dije que me voy a transformar en foca"

Yo: "Por eso, si no vez, porque no enfocas, usa los lentes" $P$ : "asch, no me entiendes, bueno, regresaré a ser pollo"

(Transcripción directa sesión 6: abril 2015)

En este fragmento se articulan tres aspectos de la relación mano, prótesis y juego, el primero es el quitar-poner que representa el uso de la prótesis, un uso que, es percibido como algo no común, que no se quiere apropiar al cuerpo y a la dinámica cotidiana, ya que, se puede quitar y poner, aun no se considera un objeto necesario e importante para la rutina diaria.

El elemento de los lentes y el juego lúdico de sonido de las palabras ${ }^{2}$ enganchan el segundo aspecto, la percepción de quiero o no quiero poner y quitar, es decir, si acepta poner algo y lo deja, es afrontar la realidad de una limitante, los lentes simbolizan dentro del juego un objeto que marca la diferencia, si los lentes eliminan la limitante de la visión, entonces la prótesis de mano equivale a eliminar la limitante de verse incompleto.

La percepción del uso de la prótesis se observa en la siguiente conversación, donde la puntualización de la demanda constante del discurso materno y del proyecto de la mano artificial, se ensamblan en el deber de aceptar dispositivo ajeno al cuerpo, aunque no se desee:

$P$ : "No me voy a poner lo que tú quieres", "no me entiendes, no quiero ponerme lo que tu decidas".

Yo: Pero tú decides, si no enfocas, ponente estos lentes. $P:$ No entiendes

Yo: Si te entiendo, que tú decides, si no enfocas, ponte unos lentes

P: -Grita ¡Bueno, si tú dices que los use, los uso!

Yo: "Ah, entonces si te molesto mucho, ¿vas a usar lo que yo quiera que uses?"

$P$ : "Asch ya me voy, en mi corcel", - el pescado que tenía puesto en el muño-emprendiendo la huida por toda la habitación.

(Transcripción directa sesión 6: abril 2015)
El juego como elemento nodal, consolida la relación de la mano y la prótesis manifestando cambios repentinos o transformaciones imposibles, por ejemplo, el ser niño que juega construye busca y hace preguntas, pero el seguir este rol pone al paciente en una posición que necesita protección, cuidados y orientación, generando actitudes reactivas, como huida, desvíos en el tema, enojo ante condiciones donde se impone un procedimiento, una necesidad o un deseo de alguien más.

\section{El juego en el discurso paterno $y$ materno}

El juego desde la perspectiva materna y paterna es diferente, como se puede observar en el siguiente fragmento:

\section{Discurso paterno:}

Padre: A escondidas me lo llevo en la bici, porque si se entera mi mujer se enoja

Yo: ¿De qué se enoja?

Alex: De que manejo la bicicleta con una mano y rápido, tiene miedo de que me caiga

Yo: Y qué pasaría si te caes

Padre: iNo se mi mujer!, ella piensa que se va a descomponer la prótesis

Yo: Y cuando vas en bici, llevas la prótesis

Ambos: No

Yo: ¿pero se llevan la prótesis?

Ambos: A veces

Yo: Y qué otra cosa no te deja hacer tu mamá,

P: Subirme a los juegos de la feria, ya tiene mucho que no vamos a las ferias

Yo: ¿Y te gustaría ir?

$P$ : Si, pero mi mama no me deja

Padre: Yo me lo llevo, le digo que me acompañe a un mandado, en lo que yo me voy al mandado, él se va a los juegos

Yo: Entonces si vas a los juegos de la feria

$P$ : Si, pero rápido.

Yo: Termino el tiempo, no vemos a la próxima

P: Oiga y lo que le platicamos, se lo va a ¿contar a alguien?

Yo: ¿A quién se lo contaría?

Padres y Paciente: -al mismo tiempo-pues a mi mamá.

Yo: ¿a su mamá?

Padre: Bueno a mi mujer, bueno es que ella me cuida mucho, tiene miedo de que me caiga, que me lastime, porque ya no veo bien, por eso manda a alguno de los niños que me acompañe.

P: Si tenemos que acompañar a mi papá a todas partes porque mi mamá se preocupa.

(Entrevista con el padre e hijo, sesión 10: mayo 2015)

\footnotetext{
2 Se utilizaron formaciones lúdicas con los sonidos de las palabras no necesariamente tienen la misma raíz de significado, pero permiten la libre asociación de ideas y de juego, por ejemplo: en foca = enfoca, enfocar, enfoques, ver bien, corregir una limitante.

MARTINEZ-TELLEZ, María, MARTINEZ-TELLEZ, Rubelia, SORIANO-PORRAS, Dulce y APAN-ARAUJO, Karla. Acompañamiento terapéutico para la aceptación o rechazo de una prótesis: Caso de paciente infantil con amputación de miembro superior izquierdo. Revista de Fisioterapia. 2019 
Se observa que para el padre el juego representa la oportunidad de hacer cosas, por ejemplo, vencer un miedo, divertirse, sin embargo, está presente el temor de que ocurra algo malo, como un accidente, o un contratiempo, es decir, el juego es una actividad para hacer y ser, pero implica un riesgo, esta percepción es una construcción del discurso materno, como el siguiente fragmento:

\section{Discurso Materno:}

Yo: ¿Su hijo ya se sube a los juegos?

Madre: No, no lo dejo... Es que para mí pasar junto a una feria, me hace sentir como cuando alguien muere. $Y$ pienso, si, el padre -sacerdote- no se hubiera tardado ese dia, mi hijo tendría su mano... Solo sé que la sensación que tengo cada vez que hay una feria, o paso por alguna, tengo ganas de pasar lo más rápido posible, y me da mucha tristeza, como cuando se muere alguien.

Yo: Y a la bicicleta, ¿se sube?

Madre: No, tampoco lo dejo, es que me entran ansias porque no veo lo que hace, se vaya a caer, si lleva la prótesis se le puede descomponer, o romper. No veo lo que hace, no sé lo que hace.

Yo, Considera que pudo haber evitado el accidente Madre: Si, si no lo hubiera dejado ir a los juegos, exigiéndole que se quedara hasta que llegara el padre, no hubiera pasado. Pero por algo pasan las cosas y diosito sabe lo que hace.

(Entrevista con la madre. Sesión 11: mayo 2015)

Los elementos relevantes en el discurso materno son el juego, el saber y el control.

El juego sea mecánico o no mecánico, representa poner en juego el cuerpo, las decisiones, donde el saber y el control se articulan para resolver la idea de que, si era posible prevenir el accidente, y el juego le interpela al discurso materno esa posibilidad de no control, de sorpresa y culpa, por lo tanto, el juego como representación contiene ideas de peligro, control, prevención, equivalente a juego-peligro, juego-no saber.

Si se observan ambos discursos el juego y el peligro son constantes, articulando la idea de prohibido, y ante lo prohibido la salida es lo clandestino, habilitado por el padre, pero sin socavar el discurso de la madre, se va a andar en bici, se sube a los juegos, se va con amigos, pero con la mancuerna de que entre ambos se están cuidado, por lo tanto, se salvan de trasgredir alguna norma o mandato.
Entonces, si el juego, saca a la luz la paradoja familiar que equivale a la idea de que un niño es un ser al que se le debe brindar protección y cuidados, pero a la vez, se espera que decida por sí mismo, como si, por él mismo tuviera el poder de saber que le conviene o no, entonces, el rechazo al juego proviene de una prohibición establecida por la relación materna, la cual se trastocada por el padre a escondidas donde el juego permite que el paciente se acerque a aquello le fue traumático. Sin embargo, dando la posibilidad de mantener un rol asignado por la relación materna que es de cuidados.

En resumen, el dispositivo clínico, muestran la representación de juego como un componente principal en la relación accidente, mama, papa, Paciente A, uso de prótesis, donde la frase el "el juego es de niñitos", es el resultado de la articulación de estos relatos que construyen verdades, tales como lo que debe hacer un niño, para la madre la relación juego- amputación, genera la anulación y prohibición completa y tacita de juego, por lo tanto un niño debe jugar pero con mayor restricción para que no se lastime.

La figura del padre activa la función de juego pero de manera clandestina, evita marcar un límite entre el discurso de la madre, dándole legalidad, pero con la posibilidad de hacer una trampa, a través del ocultamiento de la verdad: el niño juega.

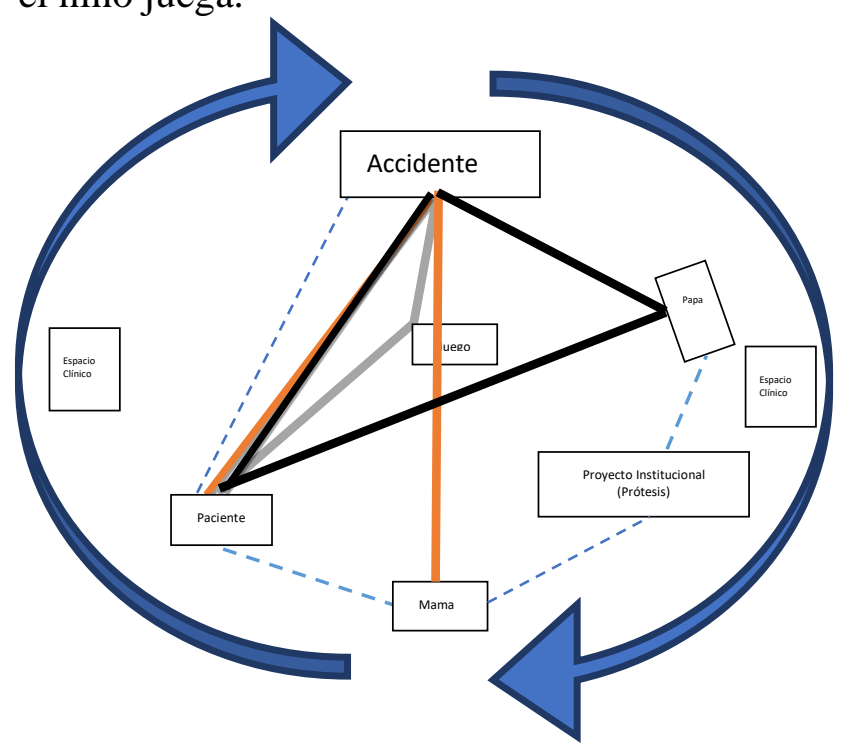

Figura 1 Esquema de Relaciones

A esta relación triangular, se suman dos elementos más, el género y el peligro de una perdida más. (Ver Figura 1) 


\section{El discurso materno y paterno entono al género}

Una vez identificada la relación del juego y la amputación, el dispositivo lúdico-narrativo se centró en crear escenarios terapéuticos que permitieran problematizar la representación: el juego es para niñitos. Esto reveló un elemento ontológico, el género - ¿yo que soy, niño o nena? - manifestando frases como:

\section{Discurso Paciente:}

Yo: Estas bien, ¿te dolió?

$P:$ No, yo aguanto, el acusarse es de nenas

Yo: ¿nenas? ¿Cómo?

P: Si es como cuando te hacen algo y vas de soplón con la maestra eso es de putos

Yo: ¿Putos?

P: Si maricas, jotos, uno tiene que aguantarse, si te la cantan, y no haces caso, eres un puto también.

Yo: ¿Cantar? ¡i Aaa!!!, i i Lalalalalala!!!!

$P$ : iii No!!!!! iii jajajaja!!! cantar, es cuando te dicen algo que te ofende y si no respondes y vas de chivo, eres un puto...El cantarla, son peleas, golpes, no esperas a acusar o platicar

Yo: ¿y cuantas has ganado?

P: En esta semana todas..

(Transcripción directa Sesión 20: julio 2015)

La percepción de genero está presente en la demostración de fuerza, potencia y virilidad, mantenerse estoico ante las cosas que duelen, si no eso significa que ser débil, el binomio femenino- debilidad, hace referencia a la manifestación de la verdad, que es la limitante de la amputación, no tener mano, sin embargo, la duda sobre si es lo que él quiere seguir haciendo o no aparece, donde lo esperado es que no exista duda, ni malestar, puesto que lo masculino equivale a fuerza y seguridad como se observa en este fragmento:

Yo: ¿Como te está yendo con las peleas?

P: Bien, pero no se ¿Por qué me peleo?

Yo: Ósea que ¿no te gustan?

$P$ : Maso, si me gustan, pero me caliento bien rápido...Que luego, ya no sé porque lo estoy haciendo...

Yo: Cuando te la cantan ¿Con que te la cantan para que te calientes?

P: Con cosas...Me dicen manita, maricon, que mi mami me hace todo, entre otras cosas.

(Transcripción directa Sesión 21: julio 2015)

Lo que sostiene el relato del paciente es el discurso paterno y materno, como se lee en este apartado:

Yo: ¿Cómo ha visto a su hijo?
Padre: Bien...Pues como lo ve usted, si es inquieto, pero su mamá no lo deja, y uno que puede hacer, antes el andaba conmigo más seguido, ahora, ni al campo lo deja...Desde chico, me seguía, me ayudaba, pero ahora, que va a poder hacer, no tiene una mano. Si me sigue, lo dejo, pero lo vigilo no vaya a hacer que se lastime y mi mujer se va a enojar.

Yo: ¿Qué se lastime?

Padre: Si que se caiga, que jale mal algo, que vaya a apoyar mal el brazo, hay que cuidarlo mucho.

Yo: ¿Y antes, lo cuidaba?

Padre: Si, pero no igual, a veces con solo ver que estaba haciendo era suficiente, pero ahora debe de andar junto a mi todo el tiempo.

Yo: Entonces, cómo a su hijo ahora lo tienen junto a usted todo el tiempo, ¿es posible que no se meta en problemas? Padre: Si se mete, pero ahi es su problema, que se les puede decir nada, porque cuando uno ya no esté con ellos -refiriéndose no solo al paciente, sino a el resto de los hijos- tendrán que solucionar las cosas y que estén junto a uno todo el tiempo, no está bien, pero le digo a mi mujer y se enoja y uno que puede hacer nada.

\section{(Transcripción directa Sesión 22: Julio 2015)}

Para el padre, el hecho de que su hijo haya perdido una mano representa, el tener que cuidarlo, existiendo una diferencia entre el antes del accidente donde el cuidado se percibía como fácil de sobrellevar, dando solo una advertencia a las cosas, posterior al accidente, el cuidado se percibe como una acto cauteloso, constante e incómodo. La preocupación se acentúa cuando se le pregunta:

Yo: ¿Qué es lo que quisiera para su hijo?

Padre: Pues lo que le queda, estudiar, es lo único, pues en donde lo van a querer, quien lo va a querer sin un mano, no pude cargar, de donde saca las fuerzas, y si va a mantener a una familia, ¿Cómo le va a hacer? Solo le queda el estudio. En el campo, de obrero, no lo van a querer y no podrá mantener a una familia.

Yo: Usted se ha enterado que su hijo se allá metido en problemas o peleas

Padre: $\mathrm{Si}$

Yo: ¿Qué piensa sobre eso?

Padre: Pues, que se les va a hacer, tu les dices no lo hagas, pero lo hacen y no puede un andar de tras de ellos todo el tiempo.

(Transcripción directa Sesión 24: Julio 2015)

Cabe a aclarar que la pregunta ¿Qué es lo que quisiera para su hijo? Estaba dirigida a saber sobre la relación entre el trabajo en el campo y la colaboración, de lo cual su respuesta arroja que la mano es percibida como una herramienta, esta idea lo posiciona en desventaja para cumplir con las funciones que le demanda su género dentro de un contexto rural. El rol de genero estructurado en la familia del Paciente es ser el sostén de una familia, ser el proveedor, el que mantiene el orden, la fuerza y el mando.

MARTINEZ-TELLEZ, María, MARTINEZ-TELLEZ, Rubelia, SORIANO-PORRAS, Dulce y APAN-ARAUJO, Karla. Acompañamiento terapéutico para la aceptación o rechazo de una prótesis: Caso de paciente infantil con amputación de miembro superior izquierdo. Revista de Fisioterapia. 2019 
Considerando que el estudio lo podría llevar a algo, pero existe incertidumbre en su futuro. Por otro lado, el discurso materno, muestra una mirada equivalente, pero con una diferencia específica, el progreso:

\section{Yo: ¿Cómo ha visto a su hijo?}

Madre: Bien... Obediente, tranquilo, hace sus tareas, ¿sin ningún problema?

Yo: ¡Aaa! ¿Sin ningún problema, cree que podría llegar a tener un problema su hijo?

Madre: Si, que lo molestan por su mano, pero yo le digo que no les haga caso, que diosito lo cuida, que le eche ganas... Es un buen niño, travieso, pero obediente, le digo que vea que me ayuda con la cocina, me acompaña al mercado, me ayuda con el quehacer, sale a correr conmigo temprano y después se va a hacer su tarea, para irse a la escuela, que yo le enseñe y le adapte cosas para que pudiera lavar los trastes, lavar la ropa, vestirse. Que no debe hacer caso, que hace muchas cosas y eso es bueno.

Yo: ¿Qué es lo que quiere para su hijo?

Madre: Que siga estudiando, el será el sostén de una familia, y tiene que estudiar para no estancarse, para progresar más que nosotros, y sin estudios como va a mantener a una familia. (Transcripción directa Sesión 25: Julio 2015)

Para la madre la representación de mantener y sostener una familia está en relación con el estudio y el progreso, sin embargo, la constate puntualización en la mayoría de las sesiones de seguimiento, es que su hijo, es obediente, tiene buenas calificaciones y que no se pelea. Haciendo manifiesta la resistencia a saber, en que se ha convertido su hijo después del accidente.

\section{La percepción de la prótesis y su uso}

El tema de la prótesis se estructura en cuatro discursos: 1) solo para realizar los ejercicios fisioterapéuticos; 2) como insignia para el reconocimiento social; 3) objeto de expectativa, y 4) como un objeto que se podía dañar con facilidad, como lo muestra la siguiente tabla:

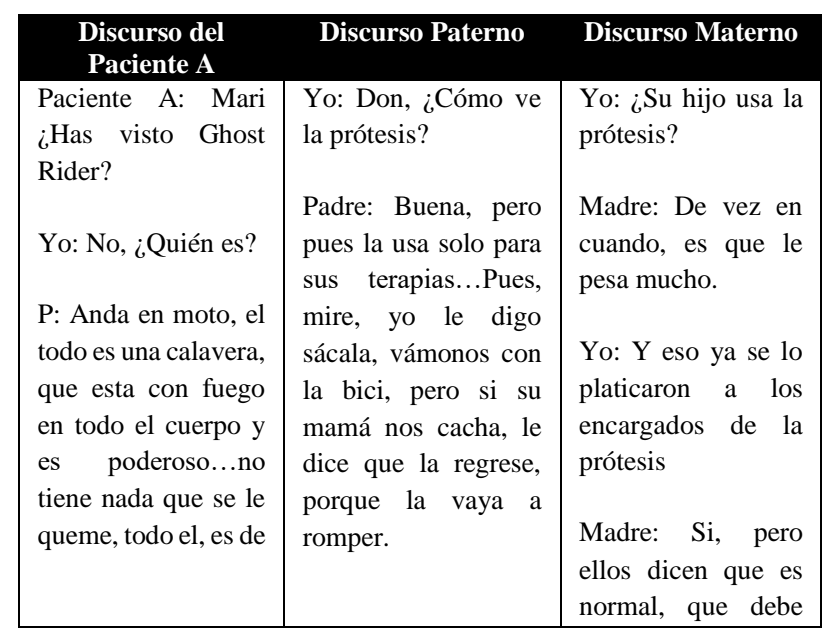

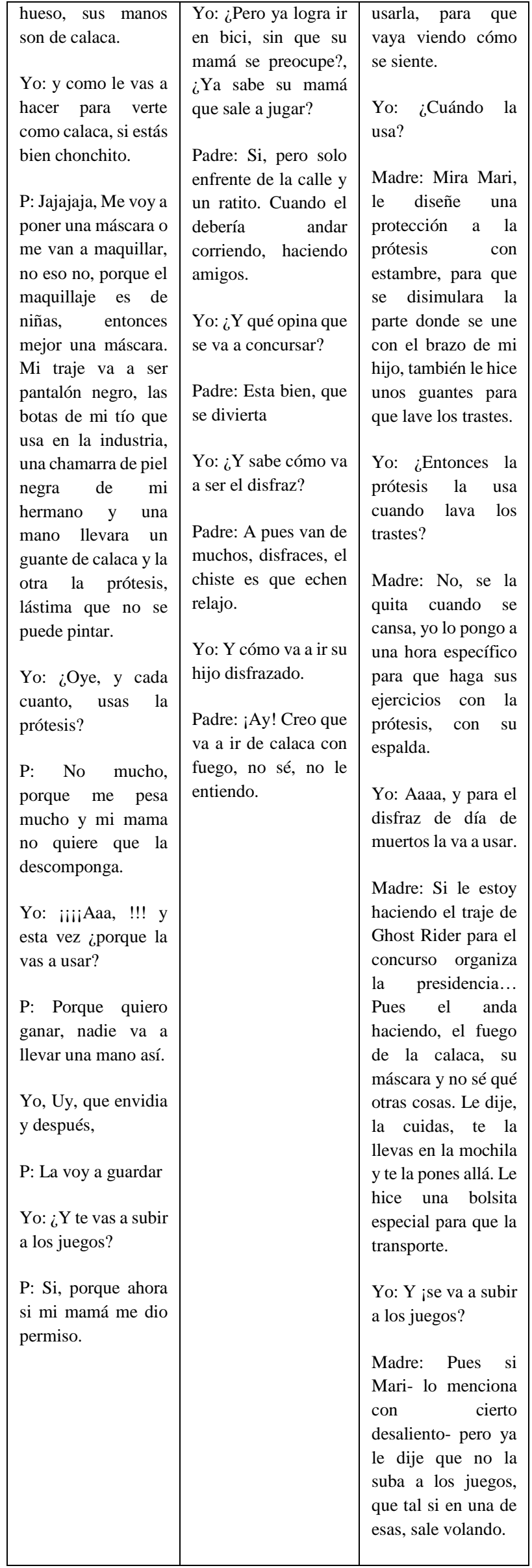

Tabla 1 Transcripción directa Sesión: víspera de día de muertos
MARTINEZ-TELLEZ, María, MARTINEZ-TELLEZ, Rubelia, SORIANO-PORRAS, Dulce y APAN-ARAUJO, Karla. Acompañamiento terapéutico para la aceptación o rechazo de una prótesis: Caso de paciente infantil con amputación de miembro superior izquierdo. Revista de Fisioterapia. 2019 
Estos fragmentos confirman como el concepto de prótesis se ha organizado entorno al cuidado y ante la previsión de no volver a perder la mano, es por ello que su uso cotidiano, la manipulación, la posibilidad de descompostura, se observe como algo negativo que se necesita controlar, sin embargo, esta percepción entraba en conflicto, puesto que el proyecto lo que demandaba era el uso constante para observar el funcionamiento, registrar anomalías, avances relacionados con el entrenamiento y el dispositivo que permitía la movilidad motora a través de las funciones cognitivas.

\section{Conclusiones}

Las metas observadas en la implementación del dispositivo lúdico-narrativo, a través del análisis de la representación del juego, permitieron que el paciente $\mathrm{A}$, transitar de conceptualizarlo como algo prohibido y amenazante a estructurar el juego como un espacio para poder construir otras alternativas de relación con su pasado, brindar la oportunidad de observar otras formas de acceder al juego, y que la clandestinidad podía ser parte de esas formas, pero no la única salida.

En el dispositivo psicoterapéutico, la clandestinidad se utilizó a favor del proceso, para facilitar la legitimidad del juego ante la madre. Lo que llevó al otorgamiento de derecho para regresar jugar, a subirse a los juegos mecánicos, a participar en las ferias del pueblo y salir con amiguitos.

Referente al género, lo que se logró identificar es que la percepción de amputación y prótesis está asociada al rol, puesto que el rechazo en el uso de la prótesis, estuvo cimentada en la funcionalidad, sostén y mantenimiento que el propio rol de varón exige, en este caso, la relación varón igual a sostén de una familia, por lo tanto, la representación de prótesis-amputación, lo estaría colocando en desventaja, en cuanto a la posibilidad de acceder al amor, al deseo, al trabajo y a él reconocimiento.

El proceso permitió brindar un orden e identificar que, en la dinámica familiar, existe una paradoja común de crianza, es decir, ser un niño es obedecer y usar algo que no se quiere, pero que se debe de usar, pero esto que se debe usar, lo tienes que cuidar, porque se puede romper, generando el conflicto, de lo uso o no lo uso, lo quiero o no lo quiero.
Por lo tanto, el uso de la prótesis se construyó entorno a observarla como un objeto de entretenimiento, de insignia y a la vez de cuidado, contrario a lo esperado por el proyecto de la Universidad Politécnica de Amozoc.

El proyecto Adaptación y Habilitación Fisioterapéutica de Prótesis Articulada Mioelectrica de Mano, esperaba que la familia y el paciente aceptaran y desear la protesis, basándose en una expectativa tacita que encontramos en los discursos institucionales: todas las personas que sufren una amputación deberían buscar el obtener un dispositivo protésico, ya que este ayudaría a mantener o mejorar el funcionamiento y la independencia de una persona, promoviendo el bienestar (OMS, 2017, 3).

Sin embargo, el caso clínico y el proceso, permitió explorar que no es suficiente saber que se tiene una necesidad para hacer todo lo posible por resolverla. Mas bien, hay elementos discursos sociales, culturales, familiares e individuales que la envisten, ya sea para hacer dudar, para decidir y hacer propio un dispositivo que sustituirá esa parte del cuerpo por el resto de la vida, un dispositivo que no se tenía previsto nunca utilizar.

Es por ello, que se propone trabajar la intervención psicoterapéutica a la par del entrenamiento fisioterapéutico para la adaptación y habilitación de una prótesis y no después, o cuando se comiencen a observar conductas o comportamientos que puedan obstaculizar el propio tratamiento físico y el uso de la prótesis.

Este trabajo en paralelo tendría el propósito de reducir el rechazo, o abandono de la prótesis y/o del entrenamiento físico, a través del trabajo en equipo que permitirá generar planes de tratamiento, que incluyan recolección de información, evaluación de las necesidades del paciente, desarrollo, implementación y evaluación del plan, es decir, potencializar el trabajo transdisciplinario (Lusiardo, 2002, 77)

El trabajo transdisciplinario permite armonizar la comunicación de los profesionales gracias a la combinación de los limites profesionales, por medio de la enseñanza y el aprendizaje de unos con otros, las tareas se basan en la experiencias formal y disciplinaria. (79). 
El abordar los casos transdiciplinaria mente tendría la ventaja de enseñar al terapeuta fisico a escuchar e identificar de manera detallada los relatos del paciente y la familia entorno al tratamiento y a la protesis con la finalidad de poder reportar, dar seguimiento y evaluar los avances del proceso de trabajo. Al mismo tiempo el psicólogo(a) podrá ir ordenando, identificando a través de una vision psicosocial los factores que estan envistiendo el tema de la adopción, adatación y convivencia con una protesis.

Lo que se busca proponer es un modelo teórico, metodológico y técnico para la escucha, intervención y problematización de la decisión de usar o no un dispositivo protésico, mediante un marco interpretativo estructurado por una vision psicosocial, que es una mirada terciaria de la realidad que permita articular e identificar el sistema de relaciones socioafectivas y el análisis de la o las representaciones que están entorno al objeto prótesis, a la percepción del cuerpo en el imaginario social y las expectativas institucionales: familia, escuela, gobierno.

En conclusión, aunque el acompañamiento psicoterapéutico culmino con el rechazo al uso de la prótesis por parte del Paciente A, este caso, permitió observar las necesidades teóricas, metodológicas y técnicas dentro de la intervención psicoterapéutica aunado a la revisión bibliográfica sobre la atención a esta población.

En pocas palabras, la importancia de desarrollar un protocolo de intervención psicoterapéutica en pacientes de 4 a 12 años permitirá, establecer el marco interpretativo, los procedimientos y las técnicas para esta población, buscando abrir un espacio que permita tomar una decision, reducir el rechazo a la protesis y favorecer la adherencia al tratamiento fisico, así como, fomentar el trabajo continuo entre los profesionales que estén involucrados en la implementación de dispositivos que sustituyan de manera parcial o total alguna parte del cuerpo.

\section{Sugerencias}

Los protocolos de intervención psicoterapéutica en personas que sufrieron una amputación, en la revisión bibliográfica de nota que solo se introduce cuando comienzan a manifestar conductas que para los tratamiento médicos o físicos los pone en peligro.
El objetivo de este estudio de caso fue dar a conocer la necesidad de trabajar antes de la amputación, durante el proceso de recuperación y entrenamiento fisioterapéutico. Por lo que se sugiere fomentar el trabajo transdisciplinario para que el paciente ya de por si angustiado por lo que le depara el futuro, tenga un espacio donde los profesionales de salud elaboren un plan de trabajo en conjunto, para apoyar $\mathrm{y}$ brindar las herramientas e información necesarias entorno a la toma de decision que implica usar o no una protesis.

\section{Referencias}

Academia Nacional de Medicina (2015). Los Amputados, un reto para el estado. Acta de Sesión. México: Autor. Recuperado de: https://www.anmm.org.mx/actas2015/Acta_SO 04032015.pdf

Caballo, V. \& Simón, M.A. (2002). Manual de Psicología Clínica Infantil y del Adolescente: Trastornos generales y Trastornos específicos. Editorial Pirámide: Madrid.

Cabrera, Rodríguez, D.S. (2012). Comunicación para el mercadeo social "La Amputación" Campañas de comunicación interna cliente: Clínica Santa Lucía (Tesis de pregrado) Universidad San Francisco de Quito. Quito

Campillo, R. M. (2019). Terapia Narrativa de Juego. Revista Haciendo Psicología. Centro de Atención Psicológica a la Familia A.C. ISSN 1870-5618

Diaz, A. J., Leal, C. C., \& Gómez, D.M. (2013) El sufrimiento de las personas amputadas. Un Enfoque etnográfico con aplicaciones psicoterapéuticas. Revista de Psicología de la Salud. 1(1), 23-44 Recuperado de: https://doi.org/10.21134/pssa.v1i1.371

Diaz, B.L., Torruco, G.U., Martínez, H.M \& Valera, R.M. (2013) La entrevista, recurso flexible y dinámico. Revista de Investigación en Educación Médica. Departamento de Investigación e Educación Médica, Facultad de Medicina, Universidad Nacional Autónoma de México, 2(7). Pp 162-167 Recuperado en: http://riem.facmed.unam.mx/sites/all/archivos/V2N um03/09_MI_LA\%20_ENTREVISTA.pdf

Flesler, A. (2011) El niño en análisis y las intervenciones del analista. Paidós: Buenos Aires. Pp. 196

MARTINEZ-TELLEZ, María, MARTINEZ-TELLEZ, Rubelia, SORIANO-PORRAS, Dulce y APAN-ARAUJO, Karla. Acompañamiento terapéutico para la aceptación o rechazo de una prótesis: Caso de paciente infantil con amputación de miembro superior izquierdo. Revista de Fisioterapia. 2019 
Font, J.I., Llaurado, S.M., Pallarés, M.A. \& García, H.F. (2016) Factores psicosociales implicados en la amputación. Revisión sistemática de la literatura. Revista de Atención Primaria, 48(3), 207-210. Recuperado de: https://www.sciencedirect.com/science/article/p ii/S0212656715002097?via\%3Dihub

Goventes, B.Y., Alba, G.C.J. \& Arias, C.A. (2016) Protocolo de actuación en la rehabilitación de pacientes amputados de miembro inferior. Revista Cubana de Medicina Física y Rehabilitación. Centro Nacional de Rehabilitación Julios Díaz González. La Habana Cuba. 8(1), 33-43.

Hernández Sampieri, R. Fernández, Colado, C. \& Baptista, Lucio, P. (2003) Metodología de la Investigación. Ed. Tercera. McGraw Hill: México. Pp 705

Lusiardo, M. (2002) Trabajo en Equipo en ámbitos de rehabilitación. Revista Salud Militar, 24(1), 77-90. Recuperado de: https://issuu.com/ dnsffaa.gub.uy/docs/toda_una_dic2002

Martínez, C.P.C., (2006) El método de estudio de caso: estrategia de la investigación científica. Revista Gestión \& Pensamiento. Universidad del Norte Barranquilla, Colombia, núm. 20. Pp 165193. Disponible en: http://www.redalyc. org/articulo.oa? $\mathrm{id}=64602005$

Martínez, T., Trujeque, L., Soriano, P \& Apan, A, (2018) Adaptación y Habilitación Fisioterapéutica en prótesis articulada mioélectrica de mano. Revista de Fisioterapia, 2(5), ISSN 2523-6849, 24-32

Meingüer, C.M de J., Martínez, T.J.L., Cuellar, H.M., Galindo, V.O., \& Rojas, C.E. (2014) Aspectos psicológicos en el paciente amputado (Tesis). Instituto Nacional de Cancerología. México.

Miranda, C. (2017) Psicoprofilaxis quirúrgicas en cirugías no conservadoras en el contexto de un tratamiento oncológico: Amputaciones y enucleaciones. Revista Medicina Infantil. Servicio de Salud Mental. Hospital de Pediatría Juan. Garrahan. 24(2), pp 139-144. Recuperad de:

http://www.medicinainfantil.org.ar/images/stories/v olumen/2017/xxiv_2_139.pdf
Moscovici, S. (2008) Psicología Social I: Influencia y cambio de actitudes. Individuos y grupos. Paidós: México. Pp.360

Organización Mundial de la Salud (2017) Normas de Ortoprotésica de la OMS: Parte 1. ISBN 978-92-4-351248-8. Ginebra: Autor. Recuperado de: https://apps.who.int/iris/bitstream/handle/10665 /259508/9789243512488-part1spa.pdf;jsessionid=FFDCD33C5F22CC5DB19 F50CCDECD883B? sequence $=1$

Potter, J. (2008). Hacer que la psicología sea relevante. Revista Interdisciplinaria de Internet: Discurso \& Sociedad. 2(1). Pp 186-200. Recuperado en: http://www.dissoc.org/ediciones/v02n01/DS2\% 281\%29Potter.html

Prótesis Avanzadas (2019) MG Latam: Acompañamiento Psicológico. Colombia. Recuperado de: https://protesismg.com/protesisy-ortesis-mg-latam/departamentos-po-mglatam/departamento-de-psicologia-po-mglatam-protesis-avanzadas/acompanamientopsicologico/

Schaefer, C.E. (2012) Fundamento de la Terapia de Juego. El Manual Moderno: México. Pp. 385

Stracalli, B. (2018) La narrativa asistida. Curso Psicología Forense especializada en niñas, niños y adolescente. Modulo III - Tema IV. Oficina de Defensoría de los Derechos de la Infancia A.C. 7

West, J. (2000) Terapia de Juego centrada en el niño. El manual Moderno: México. Pp.280
MARTINEZ-TELLEZ, María, MARTINEZ-TELLEZ, Rubelia, SORIANO-PORRAS, Dulce y APAN-ARAUJO, Karla. Acompañamiento terapéutico para la aceptación o rechazo de una prótesis: Caso de paciente infantil con amputación de miembro superior izquierdo. Revista de Fisioterapia. 2019 